\title{
Prevalencia de tabaquismo y actitud de cambio frente al hábito tabáquico en universitarios chilenos: Importancia de la formación médica
}

\author{
JOSÉ DE GRAZIA ${ }^{(1)}$, DANIELA FAIVOVICH ${ }^{(1)}$, FELIPE FALCÓN ${ }^{(1)}$, RODRIGO DÍAZ ${ }^{(1)}$, GLADYS YENTZEN ${ }^{(2)}$ \\ y SONIA KUNSTMANN ${ }^{(3)}$

\section{RESUMEN}

Introducción: El tabaquismo es el mayor determinante de problemas de salud pública. En Chile la tasa de tabaquismo en individuos entre 17 y 24 años es de 55\%. A medida que los estudiantes de Medicina avanzan en su carrera, el conocimiento acerca del tabaquismo como determinante de enfermedad aumenta. Sin embargo, los estudiantes de cursos superiores fuman más que aquellos de cursos iniciales. Objetivo: Determinar la prevalencia de tabaquismo y la actitud de cambio frente al hábito tabáquico en estudiantes de Medicina e Ingeniería, de primer y cuarto año de la Universidad de Chile. Material y método: Estudio descriptivo-transversal. Se utilizó un cuestionario autoadministrado, registrándose variables demográficas, conducta fumadora y actitud de cambio frente al hábito tabáquico. Resultados: La muestra estuvo constituida por 490 estudiantes (28,6\% mujeres). La prevalencia actual de tabaquismo fue 18,6\% (17,9\% en mujeres y 18,9\% en hombres, pns). En Medicina la prevalencia actual de tabaquismo fue 21,4\%, en Ingeniería 16,6\% $(p<0,01)$. En ambas carreras los primeros años tuvieron una tasa similar (18,2\% Medicina y 16,8\% Ingeniería, pns). Esta cifra se mantuvo en cuarto año de Ingeniería (16,3\%), pero aumentó significativamente en cuarto año de Medicina (24,3\%). Los estudiantes de Medicina tienen un comportamiento más dinámico en su conducta fumadora que los de Ingeniería. El patrón de conducta tabáquica en mujeres es potencialmente menos dañino que en hombres. Discusión: El conocimiento médico de los efectos nocivos del tabaco no desempeña un rol en disminuir las tasas de tabaquismo. Resulta necesario potenciar en las facultades de Medicina actividades preventivas relacionadas al consumo de tabaco.

Palabras clave: estudiantes de Medicina, tabaquismo, educación médica.

\section{ABSTRACT \\ PREVALENCE OF SMOKING AND ATTITUDE TOWARDS QUITTING AMONG UNIVERSITY STUDENTS: THE IMPORTANCE OF MEDICAL TRAINING}

Introduction: Smoking is the greatest determinant of public health problems. In Chile, the rate of smoking in individuals between 17 and 24 years is 55\%. As medical students advance in their studies, their knowledge of smoking as a determinant of disease increases. Nevertheless, students from more advanced class levels smoke more than those of lower levels. Objective: Determine the prevalence of smoking and the attitude towards quitting in medical and engineering students, in their first and fourth years of studies at the University of Chile. Material and method: Cross sectional descriptive study. A self-administered survey was used, recording demographic

(1) Facultad de Medicina. Universidad de Chile. Los Refugios 16.593. Lo Barnechea. Santiago. Chile. ja.degrazia@gmail.com.

(2) Escuela de Salud Pública. Facultad de Medicina. Universidad de Chile. Chile.

(3) Departamento de Enfermedades Cardiovasculares. Clínica Las Condes. Chile. 
variables, smoking behavior, and attitude towards quitting. Results: The sample consisted of 490 students $(28.6 \%$ women). The prevalence of smoking was $18.6 \%(17.9 \%$ in women and $18.9 \%$ in men, $p=n s)$. Among medical students, the prevalence of smoking was $21.4 \%$; among engineering students it was $16.6 \%(p<0.01)$. In both areas the first year students had a similar rate $(18.2 \%$ in medical students and $16.8 \%$ in engineering, $p=n s$ ). This rate was maintained in fourth year engineering students (16.3\%), but increased significantly in fourth year medical students $(24.3 \%)$. Medical students have a more dynamic attitude towards their smoking habit than engineering students. The pattern of tobacco behavior in women is potentially more harmful than in men. Discussion: Medical knowledge of the harmful effects of tobacco doesn't play a role in decreasing the rates of smoking. It is necessary to promote preventive activities related to the consumption of tobacco in medical schools.

Keywords: medical students, smoking, medical education.

\section{INTRODUCCIÓN}

Actualmente un tercio de la población mundial de 15 años o más es fumadora ${ }^{1}$. El mayor determinante de problemas de salud pública es el aumento de la mortalidad y discapacidad asociada al tabaquismo. Se proyecta un aumento de las muertes asociadas al consumo de tabaco desde 3 millones registradas en 1990 a cerca de 8,4 millones en 2020, lo cual situará al tabaco como el mayor problema de salud pública mundial ${ }^{2}$.

En Chile la tasa de tabaquismo según la Encuesta Nacional de Salud asciende a $42 \%$ ( $48 \%$ en hombres y $37 \%$ en mujeres); y en individuos entre 17 y 24 años es de 55\% (61\% en hombres y $47 \%$ en mujeres $)^{3}$. Según el Séptimo Estudio Nacional de Drogas en Población General de Chile, de Conace, la tasa de consumo de tabaco en el último mes es de 40,9\% (43\% en hombres y $39 \%$ en mujeres); en individuos entre 19 y 25 años es de $52 \%{ }^{4}$. Se dispone de estudios realizados en estudiantes universitarios: la prevalencia de tabaquismo en estudiantes de la Universidad de Concepción es de 44\% ${ }^{5}$. En el caso de estudiantes de Medicina, no debe dejar de citarse el estudio pionero de prevalencia de tabaquismo, realizado por Cruz et al en 1985, que describe un valor cercano al $36 \% \%^{6}$; otro estudio más reciente en la Universidad de Valparaíso reporta una tasa de tabaquismo en estudiantes de Medicina de 23,6\%

Los médicos, particularmente aquellos que trabajan en atención primaria, desempeñan un rol importante en educar a sus pacientes y fomentar el cese del tabaquismo. La educación acerca de este hábito y de las enfermedades relacionadas con él resulta esencial en estudiantes de Medicina, con el objetivo de otorgarles los conocimientos necesarios para saber cómo realizar intervenciones en sus futuros pacientes fumadores.

Diversos estudios internacionales demuestran que a medida que los estudiantes de Medicina progresan en su carrera, el conocimiento que poseen del tabaquismo, como determinante de enfermedad, se incrementa. De manera sorprendente, este aumento de conocimiento no se asocia a menores tasas de tabaquismo, ya que los estudiantes de Medicina de cursos avanzados generalmente fuman más que aquellos de cursos iniciales ${ }^{8-11}$.

El objetivo del presente estudio es determinar la prevalencia de tabaquismo y la actitud de cambio frente al hábito tabáquico en estudiantes de Medicina e Ingeniería, de primer y cuarto año de la Universidad de Chile. Se escogieron estas dos carreras, a fin de comparar los resultados obtenidos en ambos grupos y determinar la importancia de la formación médica en la prevalencia de tabaquismo y en la actitud de cambio que tienen los fumadores frente al hábito tabáquico.

\section{MATERIAL Y MÉTODO}

\section{Población}

Estudio descriptivo-transversal, realizado en estudiantes de primer y cuarto año de las carreras de Medicina e Ingeniería de la Universidad de 
Chile, cuya población comprende 1.471 estudiantes entre 17 y 24 años de edad. De este universo se seleccionó una muestra aleatoria, con selección proporcional por carrera, curso y sexo, de 490 estudiantes. El estudio fue llevado a cabo en septiembre de 2007.

\section{Cuestionario}

Se utilizó un cuestionario auto-administrado y anónimo, correspondiente a una adaptación del instrumento utilizado en el estudio Ricar de la Sociedad Chilena de Cardiología y Cirugía Cardiovascular ${ }^{12}$. Se registraron variables demográficas, conducta fumadora y actitud de cambio frente al hábito tabáquico. Las variables demográficas registradas fueron sexo, edad, carrera y curso del participante. En relación a la conducta fumadora, se utilizaron las definiciones establecidas por la OPS $/ \mathrm{OMS}^{13}$, que permiten clasificar a los sujetos en 4 categorías: 1) no fumadores: sujetos que nunca han fumado o han fumado menos de 100 cigarrillos en su vida; 2) fumadores diarios: sujetos que fuman uno o más cigarrillos al día; 3) fumadores ocasionales: sujetos que fuman menos de un cigarrillo al día; 4) ex fumadores: sujetos que dejaron de fumar. La prevalencia actual de tabaquismo corresponde a la suma de fumadores diarios y ocasionales, la prevalencia de vida de tabaquismo a la suma de prevalencia actual y ex fumadores. Respecto a la actitud de cambio frente al hábito tabáquico, se utilizó el Modelo de Etapas de Cambio (Prochaska y Diclemente) $)^{14-17}$, que contempla 6 etapas: 1 ) pre-contemplación: fumadores que aun no han empezado a pensar respecto al cambio, no tienen planificado cambiar su conducta; 2) contemplación: fumadores que están conscientes de algunos beneficios del cambio de conducta, han estado reflexionando sobre realizar el cambio en los próximos 6 meses; 3) preparación: fumadores que han empezado a dar algunos pasos en la dirección del cambio, buscan información, planean realizarlo en los próximos 30 días; 4) acción: la persona inició el cambio y está abocada a mantenerlo. Este período dura aproximadamente 6 meses, tiempo estimado para considerar real y estable el cambio de conducta; 5) mantención: la persona cambió de conducta hace más 6 meses y sus esfuerzos están dirigidos a adherirse a esta nueva conducta; 6) recaída: la persona logró por un período el cambio de conducta pero regresó a alguna etapa previa. E1 cuestionario fue aplicado al comienzo de una clase de asistencia obligatoria; se solicitó el consentimiento verbal antes de la aplicación.

\section{Definición de patrón de conducta tabáquica}

Con el objetivo de comprender cómo se comportan los sujetos fumadores en relación al hábito tabáquico, se realizó una caracterización de éste, que incluyó las variables prevalencia actual de tabaquismo, prevalencia de ex fumadores, etapas de cambio acción/mantención y etapa de cambio recaída. Utilizando este patrón de conducta tabáquica se realizaron comparaciones según sexo, carrera y curso.

\section{Análisis estadístico}

Se tabularon y analizaron los datos mediante el programa StatMost v 3.2 ®. Se aplicaron pruebas de hipótesis para comparar proporciones y determinar el grado de significación estadística de las relaciones observadas y se fijaron niveles de significación de la prueba en 0,05 (nivel de confianza de $95 \%$ ).

Tabla 1. Caracterización según variables demográficas de la muestra de estudiantes de primer y cuarto año de Medicina e Ingeniería de la Universidad de Chile. 2007.

\begin{tabular}{|l|c|c|c|}
\hline & Número de estudiantes & Mujeres/Hombres (\%) & Edad promedio (años) \\
\hline 1er año de Medicina & 99 & $44,4 / 59,6$ & $18,8 \pm 1,1$ \\
\hline 4to año de Medicina & 107 & $45,8 / 54,2$ & $21,9 \pm 0,9$ \\
\hline 1er año de Ingeniería & 143 & $18,2 / 81,8$ & $18,5 \pm 0,7$ \\
\hline 4to año de Ingeniería & 141 & $17,7 / 82,3$ & $21,7 \pm 1,1$ \\
\hline Total & $\mathbf{4 9 0}$ & $\mathbf{2 8 , 6} / \mathbf{7 1 , 4}$ & $\mathbf{2 0 , 3} \pm \mathbf{1 , 8}$ \\
\hline
\end{tabular}


Tabla 2. Conducta fumadora según carrera en estudiantes de primer y cuarto año de Medicina e Ingeniería de la Universidad de Chile. 2007.

\begin{tabular}{|l|c|c|c|c|}
\hline & $\begin{array}{c}\text { Prevalencia } \\
\text { Actual } \\
\text { de tabaquismo }\end{array}$ & $\begin{array}{c}\text { Prevalencia } \\
\text { de fumadores } \\
\text { diarios }\end{array}$ & $\begin{array}{c}\text { Prevalencia } \\
\text { de fumadores } \\
\text { ocasionales }\end{array}$ & $\begin{array}{c}\text { Prevalencia } \\
\text { de ex fumadores }\end{array}$ \\
\hline Medicina & $21,4 \%$ & $10,2 \%$ & $11,2 \%$ & $9,2 \%$ \\
\hline Ingeniería & $16,6 \%$ & $7,8 \%$ & $8,8 \%$ & $5,6 \%$ \\
\hline
\end{tabular}

\section{RESULTADOS}

De los 490 estudiantes encuestados, 99 $(20,2 \%)$ correspondieron a alumnos de primer año de Medicina; $107(21,8 \%)$ a alumnos de cuarto año de Medicina; $143(29,2 \%)$ a alumnos de primer año de Ingeniería y $141(28,8 \%)$ a alumnos de cuarto año de Ingeniería. El 28,6\% de la muestra fueron mujeres; la presencia femenina en Medicina fue mayor que en Ingeniería ( $43,2 \%$ y $18,0 \%$ respectivamente). El promedio de edad de la muestra fue de 20,3 (DE $1,8)$ años: 20,5 años en mujeres y 20,1 años en hombres. Ambas muestras de primer año, al igual que ambas muestras de cuarto año fueron homogéneas respecto edad. La información de variables demográficas se resume en la Tabla 1.

\section{Conducta fumadora}

La prevalencia actual de tabaquismo fue $18,6 \%$. La cifra en mujeres fue $17,9 \%$ y en hombres $18,9 \%$ (pns).

Según carrera, en Medicina la prevalencia actual de tabaquismo fue $21,4 \%$ y en Ingeniería $16,6 \%(p<0,01)$ (tabla 2). La Figura 1 muestra la tendencia según curso: ambos primeros años presentaron una prevalencia actual de tabaquismo similar (18,2\% en Medicina y $16,8 \%$ en Ingeniería, pns). Esta tasa se mantuvo en alumnos de cuarto año de Ingeniería (16,3\%), pero aumentó significativamente en alumnos de cuarto año de Medicina (24,3\%).

Analizando por separado fumadores diarios y ocasionales, se observa que Medicina tuvo una mayor prevalencia que Ingeniería en ambos casos (fumadores diarios: 10,2\% v/s $7,8 \%, \mathrm{p}<0,01$; fumadores ocasionales: $11,2 \%$ v/s $8,8 \%, p<0,01$ ) (Tabla 2). Según curso (Figura 2), la cifra fue similar en ambos primeros años, tanto en fumadores diarios como en ocasionales. En fumadores diarios, la prevalencia ascendió a $9,2 \%$ en cuarto año de Ingeniería y a $14,0 \%$ en cuarto año de Medicina $(p<0,001)$. En fumadores ocasionales, la tendencia fue a disminuir la prevalencia a $7,1 \%$ en estudiantes de cuarto año de Ingeniería y a $10,3 \%$ en estudiantes de cuarto año de Medicina $(p<0,01)$. Los estudiantes de cuarto año de Medicina tuvieron en ambos casos (fumadores diarios y ocasionales) una prevalencia mayor que los estudiantes de cuarto año de Ingeniería.

La prevalencia de vida de tabaquismo (fumadores actuales + ex fumadores) en Medicina aumentó entre el primer y el cuarto año en cerca de 10 puntos porcentuales. En cambio, en Ingeniería esta prevalencia disminuyó cerca de 5 puntos porcentuales. En otras palabras,

Tabla 3. Etapa de cambio según carrera y curso en estudiantes de primer y cuarto año de Medicina e Ingeniería de la Universidad de Chile, 2007.

\begin{tabular}{|l|c|c|c|c|c|}
\hline & $\begin{array}{c}\text { 1er año de } \\
\text { Medicina }\end{array}$ & $\begin{array}{c}\text { 4to año de } \\
\text { Medicina }\end{array}$ & $\begin{array}{c}\text { 1er año de } \\
\text { Ingeniería }\end{array}$ & $\begin{array}{c}\text { 4to año de } \\
\text { Ingeniería }\end{array}$ & Total \\
\hline Pre-Contemplación & $20,0 \%$ & $10,5 \%$ & $8,6 \%$ & $14,3 \%$ & $12,7 \%$ \\
\hline $\begin{array}{l}\text { Contemplación/ } \\
\text { Preparación }\end{array}$ & $12,0 \%$ & $44,7 \%$ & $34,3 \%$ & $60,7 \%$ & $38,9 \%$ \\
\hline Acción/Mantención & $28,0 \%$ & $31,6 \%$ & $31,4 \%$ & $17,9 \%$ & $27,8 \%$ \\
\hline Recaída & $40,0 \%$ & $13,2 \%$ & $25,7 \%$ & $7,1 \%$ & $20,6 \%$ \\
\hline
\end{tabular}




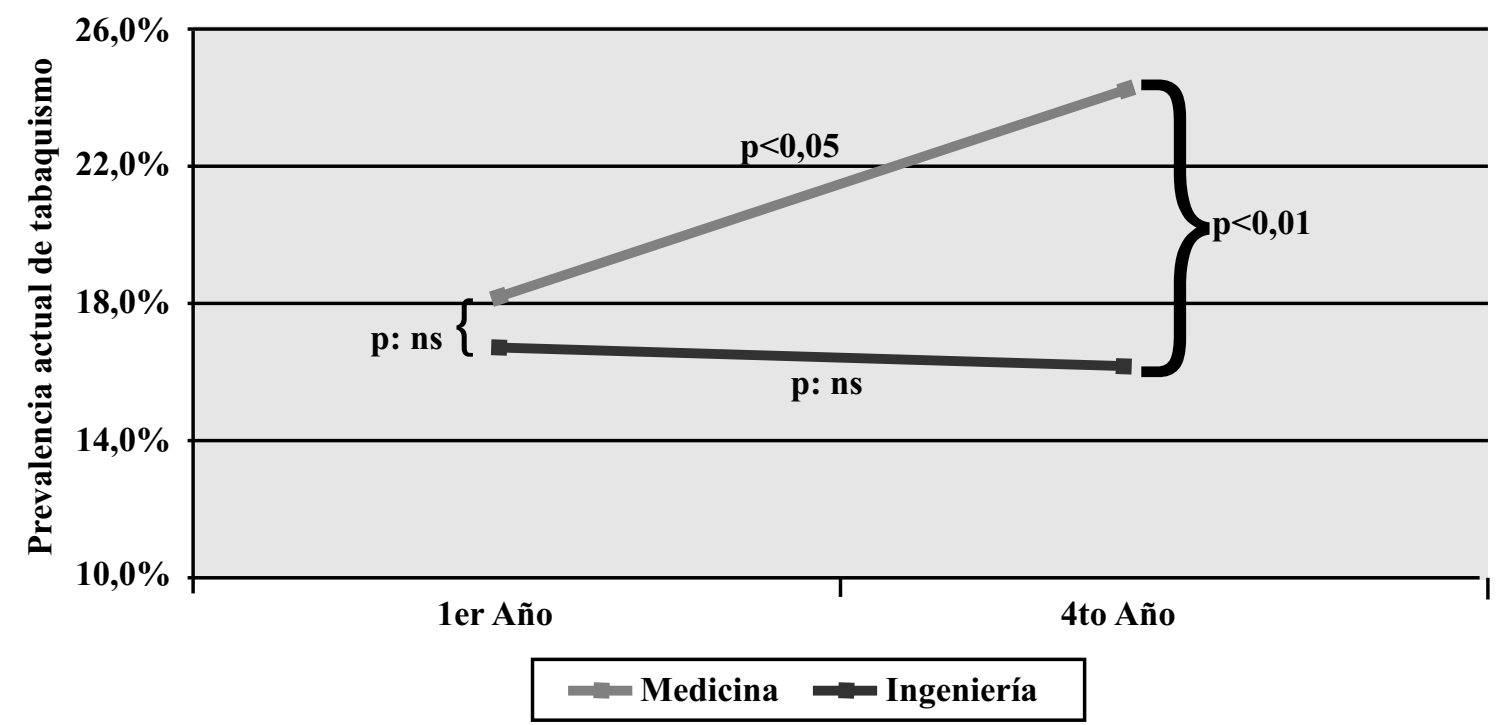

Figura 1. Prevalencia actual de tabaquismo según carrera y curso en estudiantes de primer y cuarto año de Medicina e Ingeniería de la Universidad de Chile, 2007.

en cuarto año de Medicina más de un tercio de los estudiantes ha estado expuesto al consumo de tabaco en algún momento de su vida, mientras que en cuarto año de Ingeniería sólo un quinto de los estudiantes ha fumado alguna vez.

$\mathrm{Al}$ analizar en detalle la prevalencia de vida de tabaquismo, se observa que en Medicina la prevalencia actual de tabaquismo tuvo una tendencia ascendente, aumentando desde primer a cuarto año $(18,2 \% \mathrm{v} / \mathrm{s} 24,3 \%, \mathrm{p}<0,05)$.

Además, en esta carrera los estudiantes de cuarto año presentaron también la mayor cifra de ex fumadores $(11,2 \%)$. Por otro lado, en Ingeniería la cifra de fumadores actuales se mantuvo constante entre el primer y cuarto año $(16,8 \% \mathrm{v} / \mathrm{s} 16,3 \%$, pns $)$, y la tendencia de los ex fumadores fue decreciente entre estos 2 cursos $(7,7 \% \mathrm{v} / \mathrm{s} 3,6 \%, \mathrm{p}<0,0001)$. Lo anterior demuestra que Medicina tiene un comportamiento más dinámico en relación a la conducta fumadora que Ingeniería, teniendo más individuos que se mueven entre las categorías de fumadores actuales y ex fumadores, a medida que avanzan los años de carrera. La información se resume en la Figura 3.

Actitud de cambio frente al hábito tabáquico La distribución de la muestra según etapa de cambio fue: pre-contemplación 12,7\%, contemplación/preparación $38,9 \%$, acción/mantención $27,8 \%$ y recaída $20,6 \%$ (tabla 3).

Los estudiantes de cuarto año de Medicina tendieron a encontrarse en etapas de cambio más activas (acción y mantención) que los estudiantes de primer año de la misma carrera. Por otro lado, los estudiantes de Ingeniería poseen una tendencia opuesta, encontrándose los alumnos de cuarto año en etapas de cambio más pasivas (pre-contemplación, contemplación y preparación) que los de primer año.

La etapa de recaída entre los estudiantes fumadores fue mayor en los primeros años de ambas carreras en comparación a sus respectivos cuartos años (Medicina: $40,0 \% \mathrm{v} / \mathrm{s}$ $13,2 \%, p<0,001$; Ingeniería: $25,7 \% \mathrm{v} / \mathrm{s} 7,1 \%$, $\mathrm{p}<0,0001)$. Además, la recaída fue más frecuente en Medicina que en Ingeniería (Tabla $3)$.

\section{Patrón de conducta tabáquica. Comparación según sexo}

Si bien la prevalencia de fumadores actuales en mujeres y hombres fue similar $(17,9 \% \mathrm{v} / \mathrm{s}$ $18,9 \%$, pns), ambos sexos poseen un patrón de conducta tabáquica diferente. El patrón femenino 
es potencialmente menos dañino que el masculino: las mujeres tienden a ser con mayor frecuencia fumadoras ocasionales, poseen una mayor proporción de ex fumadoras y fumadoras en etapas de cambio de acción/mantención y presentan menor recaída que los hombres (Figura 4).

\section{Comparación según carrera y curso}

El patrón de conducta tabáquica de ambos primeros años no presenta diferencias en la prevalencia de fumadores actuales, presentando incluso la misma distribución según el tipo de fumador actual (diario/ocasional). Ambos primeros años tampoco difieren en la proporción de ex fumadores ni en la proporción de fumadores en etapas de cambio acción/mantención. La etapa de recaída es más prevalente en los estudiantes de primer año de Medicina que en los estudiantes de primer año de Ingeniería, hecho que también ocurre en cuarto año. En resumen, el patrón de conducta tabáquica de los primeros años es similar entre ambas carreras. Esto no ocurre al comparar los cuartos años, que presentan un patrón de conducta tabáquica diferente entre sí. Sólo la distribución según tipo de fumador actual (diario/ocasional) es similar. La información se resume en la Figura 5.

Fumadores diarios

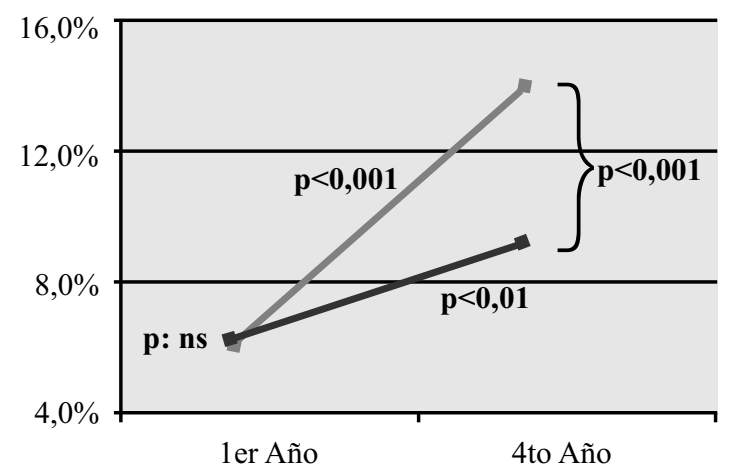

\section{DISCUSIÓN}

Determinar la prevalencia de tabaquismo y la actitud de cambio frente al hábito tabáquico en población universitaria resulta fundamental para realizar prevención a edades tempranas. Los fumadores jóvenes constituyen un grupo creciente, fumando cada vez en forma más precoz, por lo tanto hay que aplicar medidas para fomentar la abstención del tabaco en este grupo.

La prevalencia actual de tabaquismo en nuestro estudio fue $18,6 \%$, valor significativamente menor que la tasa reportada para el mismo rango etario en la Encuesta Nacional de Salud y en el Séptimo Estudio Nacional de Drogas en Población General de Chile, de Conace $\left(55 \%\right.$ y $52 \%$ respectivamente) ${ }^{3-}$ ${ }^{4}$. Lo anterior resulta concordante con resultados descritos en la literatura extranjera ${ }^{11}$, donde las cifras de tabaquismo en universitarios son también muy inferiores a las halladas en encuestas dirigidas a población general. Si comparamos nuestros resultados con los encontrados por otros autores nacionales en universitarios ${ }^{5}$, nuestras cifras resultan menores. En estudiantes de Medicina $^{7}$ la prevalencia de tabaquismo resulta ser similar.

Las mujeres poseen un patrón de conducta

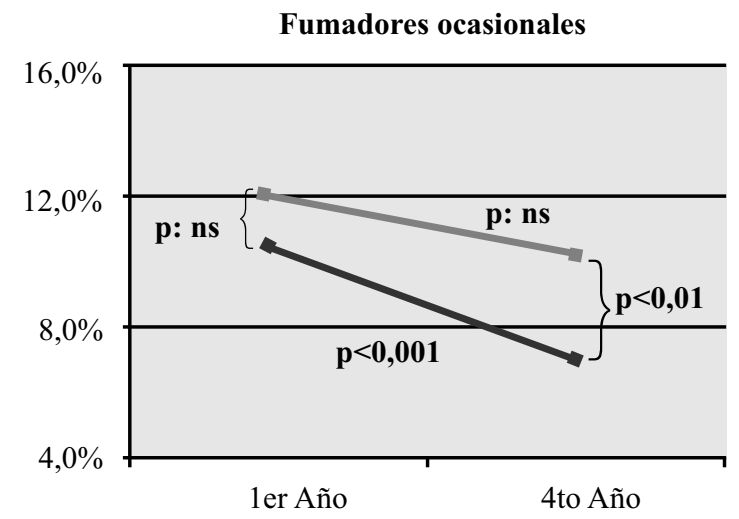

$=$ Medicina $=$ Ingeniería

Figura 2. Prevalencia de fumadores diarios y ocasionales según carrera y curso en estudiantes de primer y cuarto año de Medicina e Ingeniería de la Universidad de Chile. 2007. 


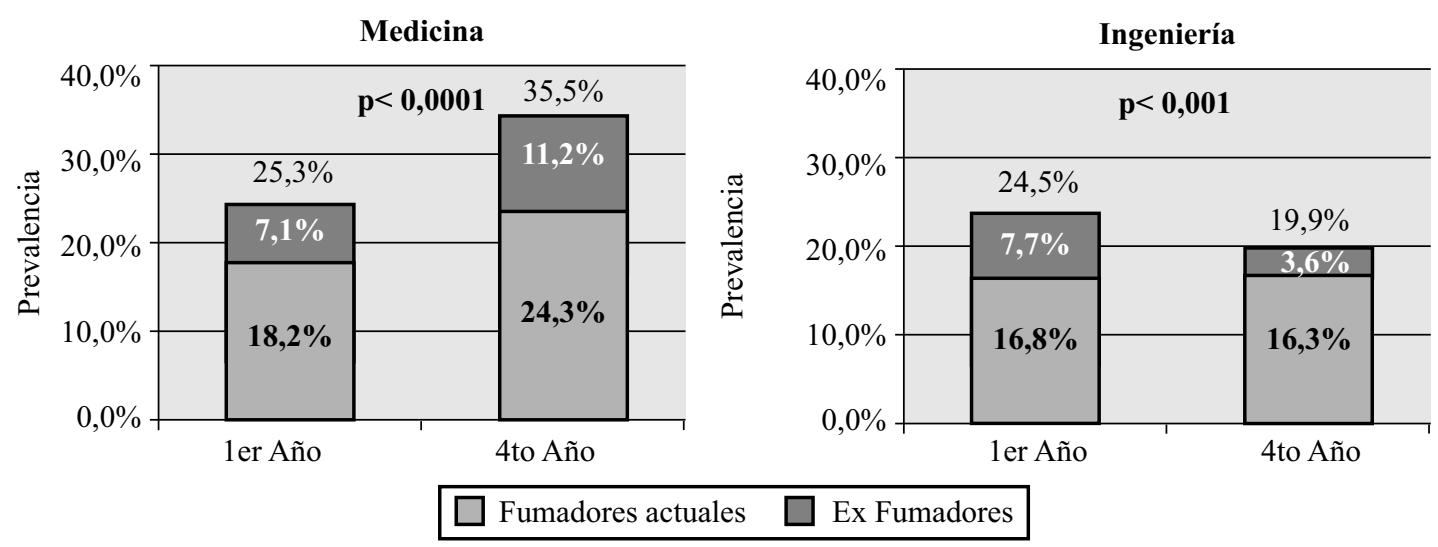

Figura 3. Prevalencia de vida de tabaquismo (fumadores actuales + ex fumadores) según carrera y curso en estudiantes de primer y cuarto año de Medicina e Ingeniería de la Universidad de Chile. 2007.

tabáquica potencialmente menos dañino que los hombres. A pesar de que la prevalencia de fumadores actuales en ambos sexos fue similar, las mujeres tienden a ser con mayor frecuencia fumadoras ocasionales, poseen una mayor proporción de ex fumadoras y fumadoras en etapas de cambio de acción/mantención y presentan menor recaída que los hombres.

Cabe destacar que los alumnos de Ingeniería y Medicina poseen características similares: se requiere un puntaje semejante en la Prueba de Selección Universitaria para la admisión en ambas facultades, ambas son carreras del área científica y ambas poseen una carga académica que puede ser homologable. Todo esto hace que los estudiantes de Ingeniería constituyan un grupo de referencia en relación al cual se puede comparar los resultados obtenidos en Medicina, considerándolos como una población control.

Al comparar los valores obtenidos en los primeros años, se observa una situación semejante respecto a la prevalencia actual de tabaquismo, incluso al analizar por separado los fumadores diarios y ocasionales. El patrón de conducta tabáquica de ambos primeros años es similar. Esto probablemente se explica por el breve lapso de estudios universitarios que llevan los estudiantes de primer año, haciendo que ambos grupos puedan ser considerados homologables.

Los estudiantes de cuarto año de Medicina presentaron la mayor tasa de tabaquismo en nuestro estudio, siendo ésta significativamente mayor que la de los estudiantes de primer año de la misma carrera; esto no ocurre en Ingeniería. Cuarto año de Medicina presentó además una mayor cantidad de sujetos en etapa recaída que cuarto año de Ingeniería. Estos hallazgos sugieren que el conocimiento médico acerca de los efectos nocivos del tabaco no desempeña un rol en disminuir las tasas de tabaquismo. Lo anterior pone de manifiesto que la Facultad de Medicina no estaría ejerciendo una influencia positiva en disminuir la prevalencia del tabaquismo, sino más bien estaría determinando que los estudiantes de Medicina de cursos avanzados fumen más que aquellos de cursos iniciales. Estos hallazgos resultan compatibles con lo descrito en la literatura internacional ${ }^{8-11}$. Para entender el porqué de estos hallazgos, se necesitarán nuevos estudios que permitan conocer las posibles causas (ejemplo: presencia de docentes fumadores, de un ambiente permisivo, etc.).

Medicina tiene un comportamiento más dinámico en relación a la conducta fumadora que Ingeniería, teniendo más individuos que se mueven entre las categorías de fumadores actuales y ex fumadores, a medida que avanzan los años de carrera. Dicho de otro modo, existen individuos iniciando el hábito tabáquico al mismo tiempo que otros individuos están dejando de fumar. Estos hallazgos ponen de manifiesto que en este grupo las estrategias de 
prevención deben también dirigirse a los individuos no fumadores, para evitar que se inicien en el consumo de tabaco.

El modelo de etapas de cambio de Prochaska y Diclemente resulta fundamental en el desarrollo de intervenciones de salud pública para la cesación del hábito tabáquico, pues utiliza la conducta y la intención del individuo para caracterizar su disposición a dejar de fumar ${ }^{14-17}$. Actualmente, muchas estrategias de intervención van dirigidas a la etapa de preparación. Sin embargo, la literatura internacional demuestra que la mayoría de los individuos se distribuyen en las etapas de precontemplación y contemplación, lo cual resulta en una disparidad entre el tipo de fumador y la intervención que en él se aplica ${ }^{14}$. Cifras nacionales muestran que la mayoría de los sujetos se encuentra en etapa de mantención ${ }^{17}$. Existe consenso en que sólo intervenciones apropiadas para cada etapa resultan eficaces en disminuir las tasas de tabaquismo en la población fumadora.

En nuestro estudio la mayoría de los sujetos está en las etapas contemplación/preparación. Además, los estudiantes de cuarto año de Medicina tienden a encontrarse en etapas de cambio más activas (acción y mantención) que

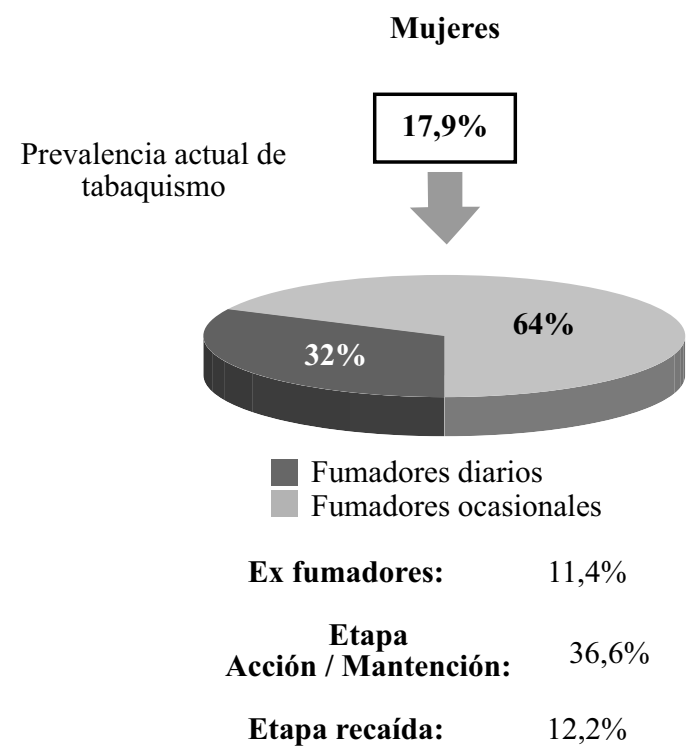

los estudiantes de primer año de la misma carrera. En otras palabras, los estudiantes de cuarto año de Medicina, pese a tener cifras más altas de tabaquismo, están más dispuestos a descontinuar el hábito tabáquico. Por otro lado, los estudiantes de Ingeniería poseen una tendencia opuesta, encontrándose los alumnos de cuarto año en etapas de cambio más pasivas (pre-contemplación, contemplación y preparación) que los de primer año.

En relación a las limitaciones de nuestro estudio, es importante señalar que por tratarse de la aplicación de un cuestionario autoadministrado, los participantes fueron voluntarios, lo que puede suponer la presencia de sesgo de selección. Además, el cuestionario utilizado corresponde a la adaptación de un instrumento preexistente, no habiéndose realizado un nuevo proceso de validación. Finalmente, la mayor limitante de nuestro estudio es que es de tipo transversal y no prospectivo. Resultaría interesante realizar un estudio prospectivo en estudiantes de Medicina chilenos, que permita evaluar la evolución de la prevalencia de tabaquismo a medida que éstos progresan en su carrera, para corroborar los hallazgos descritos en nuestro estudio.

Podemos concluir que la formación sobre

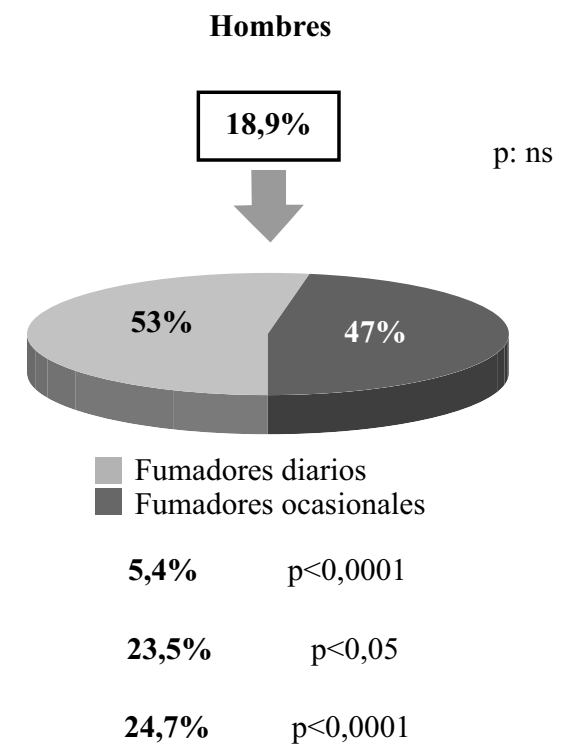

Figura 4. Patrón de conducta tabáquica según sexo en estudiantes de primer y cuarto año de Medicina e Ingeniería de la Universidad de Chile. 2007. 
Patrón de conducta tabáquica en estudiantes de primer año según carrera

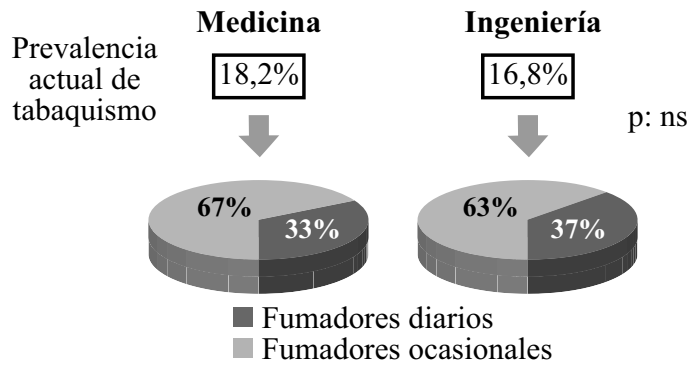

$\begin{array}{lrrr}\text { Ex fumadores } & 7,1 \% & 7,7 \% & \text { p: ns } \\ \begin{array}{l}\text { Etapa Acción/ } \\ \text { Mantención }\end{array} & 28,0 \% & 31,4 \% & \text { p: ns } \\ \begin{array}{l}\text { Etapa recaída } \\ 40,0 \%\end{array} & 25,7 \% & \text { p }<0,01\end{array}$

Patrón de conducta tabáquica en estudiantes de cuarto año según carrera.

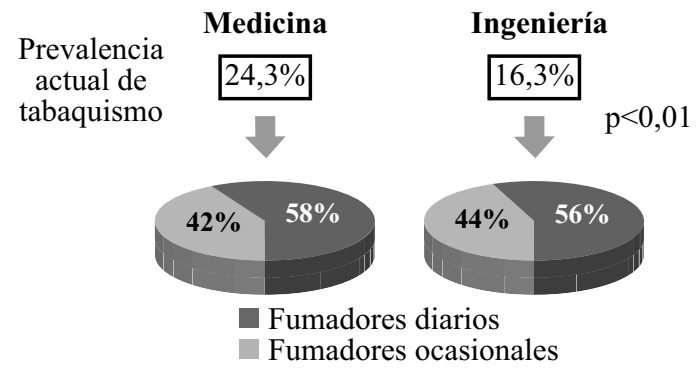

Ex fumadores $11,2 \%$
$\begin{aligned} & \text { Etapa Acción/ } \\ & \text { Mantención }\end{aligned}$
$\begin{aligned} & \text { Etapa recaída } \\ & 13,2 \%\end{aligned}$

$3,6 \% \quad \mathrm{p}<0,0001$

$17,9 \% \quad \mathrm{p}<0,05$

$7,1 \% \quad \mathrm{p}<0,01$

Figura 5. Patrón de conducta tabáquica según carrera y curso en estudiantes de primer y cuarto año de Medicina e Ingeniería de la Universidad de Chile. 2007.

tabaquismo que reciben los estudiantes de Medicina no es adecuada. El mejor consejo que puede dar un médico es no fumar él. Este concepto no se transmite en la educación que la Facultad de Medicina aporta a los futuros médicos, sobre todo si éstos son fumadores. Coincidimos con otros autores ${ }^{8-11}$ en señalar que la formación médica actual no logra disminuir el porcentaje de fumadores, sino más bien favorece que las tasas de tabaquismo en los estudiantes de Medicina se incrementen a medida que éstos progresan en sus años de carrera. Los conocimientos acerca de los efectos perjudiciales del tabaco tienen escaso impacto sobre la conducta de los estudiantes. Por ello, es adecuado incorporar durante el pregrado formación específica respecto al tabaquismo, asociado a programas de prevención del hábito. Resulta necesario potenciar en las facultades de Medicina las actividades formativas e informativas específicas en torno al consumo de tabaco y desarrollar todos los medios posibles para disminuir este problema de salud.

\section{REFERENCIAS}

1. COLLISHAW NE, LOPEZ AD. The tobacco epidemic: a global public health emergency. Tobacco Alert. Geneva: World Health Organization, 1996.

2. MURRAY CJL, LOPEZ AD. Alternative projections of mortality and disability by cause 1990-2020: Global Burden of Disease Study. Lancet 1997; 349: 1498-504.

3. MINISTERIO DE SALUD, Chile. Encuesta Nacional de Salud. 2003.

4. MINISTERIO DEL INTERIOR, Consejo Nacional para el Control de Estupefacientes, Chile. Séptimo Estudio Nacional de Drogas en Población General de Chile. 2006.

5. SOTOMAYOR H, BEHN V, CRUZ P et al. Tabaquismo en académicos, no académicos y estudiantes de la Universidad de Concepción. Rev Med Chile 2000; 128(9): 977-84.

6. CRUZ E, LISBOA C, AGUIRRE C, PERTUZE J. Estudio longitudinal del hábito de fumar en estudiantes de la Universidad Católica - Característica del hábito en los ingresos de 1982 a 1984. Rev Med Chile 1985; 113: 1231-39.

7. MC COLL P, AMADOR M, AROS J, LASTRA A, PIZARRO C. Prevalencia de factores de riesgo de enfermedades crónicas no transmisibles en estudiantes de medicina de la Universidad de Valparaíso. Rev Chil Pediatr 2002; 73(5): 478-82.

8. RICHMOND R. Teaching medical students about tobacco. Thorax 1999; 54: 70-78.

9. RICHMOND R, KEHOE L. Smoking behaviour and attitudes among Australian medical students. Med Educ 1997; 31: 169-76.

10. KNOPF A, WAKEFIELD J. Effect of medical education on smoking behaviour. Br J Prev Soc Med 1974; 28: 246-51.

11. NERÍN I, GUILLEN D, MAS A, CRUCELAEGUI A. Evaluación de la influencia que ejerce la Facultad de Medicina en los futuros médicos respecto al tabaquismo. Arch Bronconeumol 2004; 40(8): 341-7.

12. KUNSTMANN S, LIRA MT, MOLINA JC, MERUANE J, GUARDA E, MARCHANT E ET AL. 
Riesgo de presentar un evento cardiovascular a 10 años en personas sanas: Proyecto RICAR. Rev Chil Cardiol 2004; 23(1):13-20.

13. ORGANIZACIÓN PANAMERICANA DE LA SALUD / ORGANIZACIÓN MUNDIAL DE LA SALUD. Guías para el Control y Monitoreo de la Epidemia Tabáquica. 1996.

14. VELLICER WF, FAVA JL, PROCHASKA JO, ABRAMS DB, EMMONS KM, PIERCE JP. Distribution of smokers by stage in three representative samples. Prev Med 1995; 24: 401-11.

15. VELICER WF, ROSSI JS, PROCHASKA JO, DICLEMENTE CO. A criterion measurement model for health behavior change. Addictive Behaviors 1996; 21(5): 555-84.

16. PROCHASKA JO, VELICER WF. The transtheoretical model of health behaviour change. Am J Health Promot 1997; 12(1): 38-48.

17. LIRA MT, KUNSTMANN S, CABALLERO E, GUARDA E, VILLAROEL L, MOLINA JC. Prevención cardiovascular y actitud de cambio frente a los factores de riesgo: un análisis crítico del estado actual. Rev Med Chile 2006; 134: 223-30.

Recepción: 26 junio 2009 Aprobación: 3 septiembre 2009

Usted puede comentar éste y otros artículos publicados en la Revista Chilena de Salud Pública, enviando un correo electrónico a revistasp@med.uchile.cl 